\title{
Fluid-particle energy transfer in spiral jet milling
}

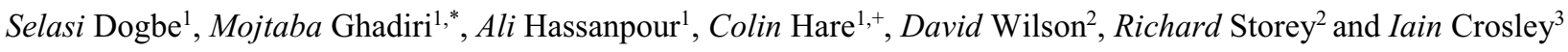 \\ ${ }^{1}$ School of Chemical and Process Engineering, University of Leeds, Leeds LS2 9JT, UK \\ ${ }^{2}$ AstraZeneca, Macclesfield SK10 2NA, UK \\ ${ }^{3}$ Hosokawa Micron, Runcorn WA7 3DS, UK
}

\begin{abstract}
Spiral jet milling is a size reduction process driven by the fluid energy of high velocity gas jets. Inter-particle and particle-wall interactions are responsible for size reduction. The process is energy intensive, but inefficient. The underlying mechanisms for size reduction in the mill are also not very well understood. The optimum grinding conditions are still currently found by trial and error experimentation.

In this work, the Discrete Element Method coupled with Computational Fluid Dynamics is used to investigate the effects of different parameters on the particle collisional behaviour in a spiral jet mill. These include the particle concentration in the grinding chamber, the particle size, and the fluid power input. We report on our work analysing the efficiency of energy transfer and how it can be improved by changing the milling conditions and particle properties.
\end{abstract}

\section{Introduction}

The spiral jet mill is a size reduction equipment capable of reducing particulate solids to micron sizes with narrow size distributions. This makes it particularly suitable for industries such as paint, food, and pharmaceuticals, where such product specifications are commonly required. For example, in the pharmaceutical industry the dissolution of the active pharmaceutical ingredient (API) in the target being, content uniformity of the API in a tablet dosage form, and drug delivery by dry powder inhalers all require control of the particle size.

The spiral jet mill functions using solely high-pressure gases to induce particle breakage and classification of the milled material within the grinding chamber. The spiral jet mill contains two gas inlets: the injector gas and grinding gas inlets. The injector gas is used to propel the feed material into the grinding chamber of the mill. The grinding gas creates a circular motion of fluid in the grinding chamber which entrains the entering feed material. At this point there are two competing forces acting on the particles: the centrifugal and drag forces. When the particle is large, the centrifugal force outweighs the drag force, and particles are pushed towards the mill wall, where they experience inter-particle and particlewall interactions that lead to size reduction. When the particles are reduced to a certain size, known as the cut size, the drag force becomes the dominating force. This causes particles to be pulled towards the central outlet and escape from the grinding chamber.

Spiral jet milling is a highly inefficient process, with only as little as $2 \%$ of the supplied energy being utilised in particle breakage [1]. Moreover the performance is strongly dependent on the mill design, affecting the relative contributions of fragmentation and surface chipping to the size reduction. The use of numerical simulations allows the role of important parameters to be identified and enables the cost efficient design and optimisation of the milling process. Previous researchers have simulated the spiral jet mill to investigate aspects of the process ranging from the velocity field to particle classification [2-5].

In this work the Discrete Element Method (DEM) is used coupled with Computational Fluid Dynamics (CFD) to analyse particle dynamics. The Hosokawa Alpine Aeroplex Spiral Jet Mill 50AS was used as the simulation geometry.

\section{Method}

Using $\mathrm{CAD}$, the grinding chamber of the Hosokawa Alpine Aeroplex Spiral Jet Mill 50AS was drawn with the feed inlet and injector nozzle attached. Only this section of the mill was used in the simulation as this is the primary location of all milling activity. The chamber has a nominal diameter of $50 \mathrm{~mm}$, and four evenly spaced grinding nozzles tangentially mounted to the wall.

DEM calculations were carried out using EDEM 2.7 (DEM Solutions, UK). ANSYS Fluent (ANSYS, USA) was used for CFD calculations. Particle properties common to all conducted simulation cases are listed in Table 1. The k-epsilon $(\mathrm{k}-\varepsilon)$ turbulence model was used for all simulations.

\footnotetext{
* Corresponding author: M.Ghadiri@,leeds.ac.uk

${ }^{+}$Current address: Department of Chemical and Process Engineering, University of Surrey, Guildford GU2 7XH, UK
} 
Table 1. Particle material properties

\begin{tabular}{|c|c|}
\hline Poisson's ratio & 0.35 \\
\hline Density $\left(\mathrm{kg} / \mathrm{m}^{3}\right)$ & 1525 \\
\hline Coefficient of restitution & 0.5 \\
\hline Coefficient of sliding friction & 0.5 \\
\hline Coefficient of rolling friction & 0.01 \\
\hline
\end{tabular}

Table 2. Number $\%$ of each particle size in the simulation

\begin{tabular}{|c|c|c|c|c|c|c|}
\hline \multicolumn{2}{|c|}{$\begin{array}{c}\text { Particle size } \\
(\boldsymbol{\mu m})\end{array}$} & 10 & 20 & 50 & 75 & 100 \\
\hline \multirow{2}{*}{$\begin{array}{c}\text { Number } \\
\%\end{array}$} & $\begin{array}{c}\text { Case } \\
\mathbf{1}\end{array}$ & 62.9 & 31.4 & 5.03 & 0.60 & 0.07 \\
\cline { 2 - 7 } & $\begin{array}{c}\text { Case } \\
\mathbf{2}\end{array}$ & 20 & 20 & 20 & 20 & 20 \\
\hline
\end{tabular}

\subsection{Collisional Energy}

The collisional energy in the spiral jet mill was investigated for two cases containing 10,000 particles: one with a milled product size distribution of particles in the chamber, and one with an equal number of different sized particles. The size and number of each particle in the simulations are given in Table 2.

Both simulation cases were run using the Hertz-Mindlin contact model.

\subsection{Effect of Particle Concentration}

The spiral jet mill was simulated at grinding and injector pressures of 4.0 bar, with three different numbers of particles: 10,000, 100,000, and 1,000,000. The particle size distribution followed Case 1 shown in Table 2. The number of collisions and the dissipated energy of interparticle and particle-wall collisions were investigated in each case. These simulations were carried out using the Hertz-Mindlin contact model.

Under the same grinding conditions, the relative velocity distribution of particles was investigated in cases containing 2,500, 5,000 and 10,000 monosized (425 $\mu \mathrm{m})$ particles. The elasto-plastic-adhesive contact model developed by Pasha et al. [6] was used for these simulations, but adhesion was switched off.

\subsection{Effect of Injector and Grinding Nozzles Gas Pressures}

With a number of 2,500, 5,000 and 10,000 monosized $(425 \mu \mathrm{m})$ particles, the spiral jet mill was simulated with various grinding and injector pressures (see Table 3 ). The Pasha et al. model [6] was used for these simulations.
Table 3. Grinding and injector pressure combinations

\begin{tabular}{|c|c|}
\hline \multicolumn{2}{|c|}{ Pressure (barg) } \\
\hline Grinding & Injector \\
\hline 1.0 & 1.0 \\
\hline 2.0 & 2.0 \\
\hline 3.0 & 3.0 \\
\hline 4.0 & 4.0 \\
\hline 5.0 & 5.0 \\
\hline 6.0 & 6.0 \\
\hline
\end{tabular}

\section{Results}

\subsection{Collisional Energy}

It is found that the dissipated energy between normal inter-particle collisions is greater than that of particle-wall collisions, indicating that the former contributes more towards size reduction than the latter (Fig. 1). This was found to be the case for all collisions except a $10 \mu \mathrm{m}$ particle colliding with a $50 \mu \mathrm{m}, 75 \mu \mathrm{m}$ and $100 \mu \mathrm{m}$ particle. This was due to the fact that the contact area between the $10 \mu \mathrm{m}$ particle and the aforementioned particle sizes is smaller than that of the contact area between the $50 \mu \mathrm{m}, 75 \mu \mathrm{m}$ and $100 \mu \mathrm{m}$ particles and the wall. The larger contact area means there will be a greater amount of deformation, and consequently dissipated energy. Another observed trend is the amount of dissipated energy increases as the particle size increases.

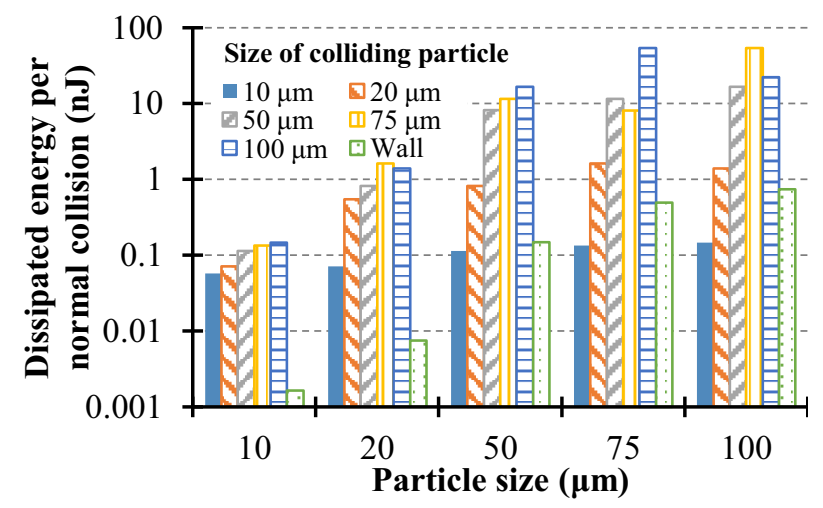

Fig. 1. Dissipated energy per normal inter-particle and particlewall collisions

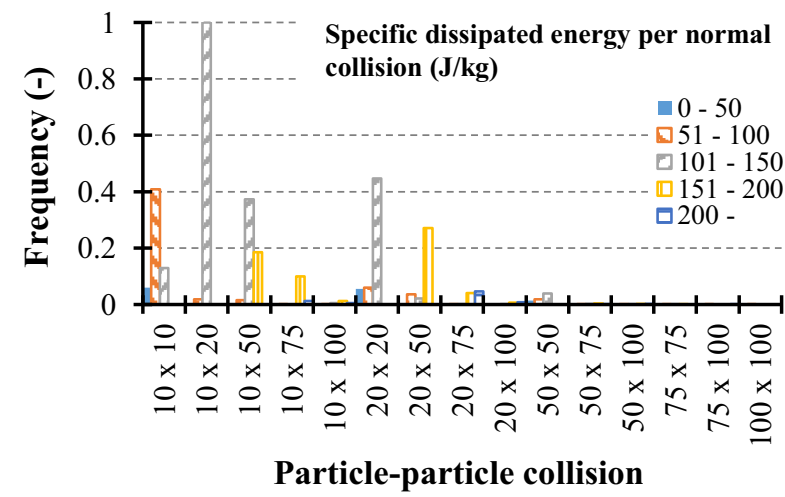

Fig. 2. Specific dissipated energy per collision and its frequency for every possible inter-particle collision (Case 1) 


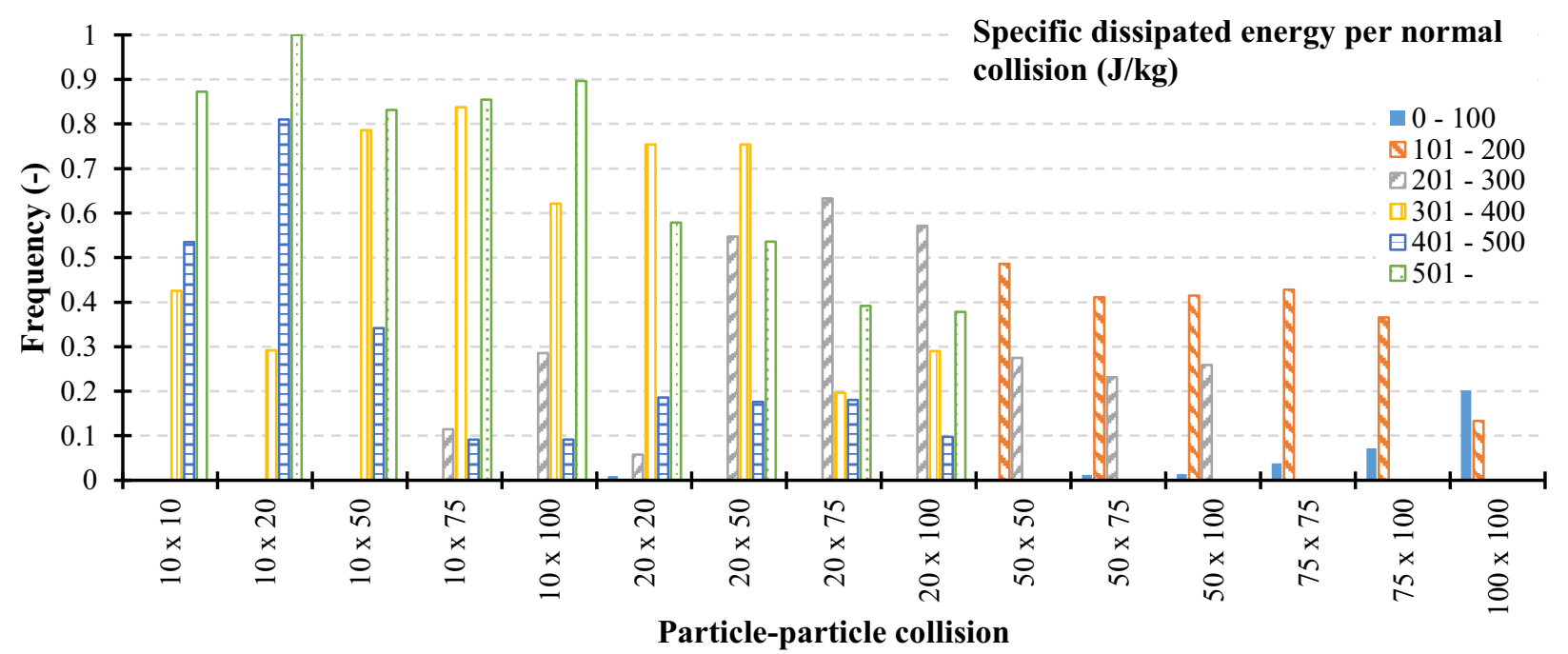

Fig. 3. Specific dissipated energy per collision and its frequency for every possible inter-particle collision (Case 2)

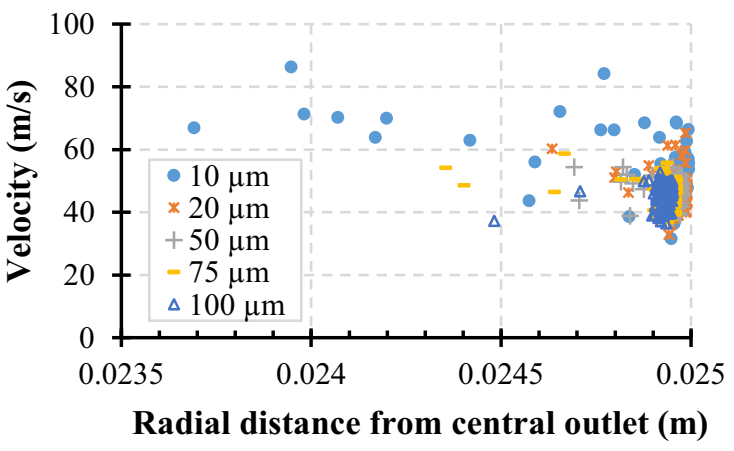

Fig. 4. Velocity magnitude and distance from central outlet of the different particle sizes ( 2000 of each particle size)

Analysis of the various possible inter-particle collisions in terms of their frequency and specific dissipated energy per collision shows that collisions between 10 and $20 \mu \mathrm{m}$ particles have a much higher frequency compared to all other possible combinations, with a dissipated energy between $101-150 \mathrm{~J} / \mathrm{kg}$ (Fig. 2). Therefore collisions with 10 and $20 \mu \mathrm{m}$ particles have a higher rate of breakage than other possible collisions. This also suggests there exists an optimum size ratio for the rate of breakage in terms of inter-particle collisions.

When there is an equal number of each particle size (Case 2 ), it is again seen that collisions between 10 and $20 \mu \mathrm{m}$ particles have a higher frequency and dissipated energy per collision than the other possible collisions (Fig. 3), indicating that this behaviour is not solely due to the size distribution.

Looking at the radial position of the different sized particles, there exists a size segregation of the particles as they circulate around the outer wall of the spiral jet mill (Fig. 4).

From the relative collisional velocity distribution of the different particle sizes in the mill with other particles (Fig. 5), it can be seen that 10 and $20 \mu \mathrm{m}$ particles have a wider velocity distribution with generally higher relative velocity magnitudes. This combined with the relative positions of the particles indicates why there may exist an optimum size ratio. The design and operating fluid dynamics undoubtedly influence this size ratio.

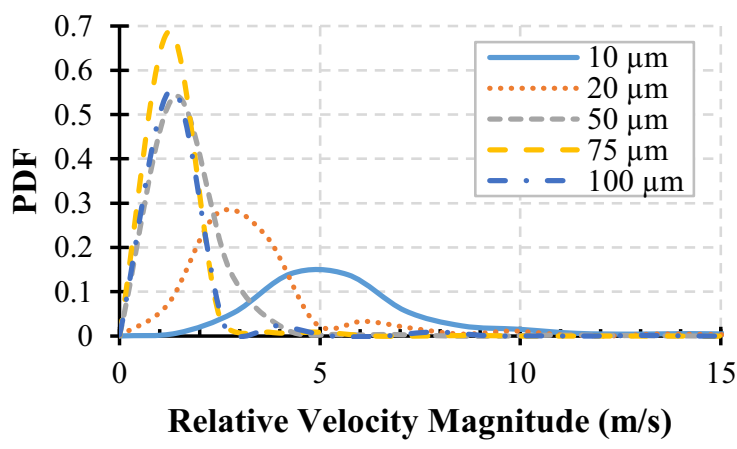

Fig. 5. Velocity distribution of different particle sizes

\subsection{Effect of Particle Number}

Increasing the number of particles in the spiral jet mill influences the proportion of the total dissipated energy due to inter-particle and particle-wall collisions (Table 4). With $10^{4}$ particles the majority of collisions and dissipated energy are due to particle-wall collisions. When the number of particles is increased to $10^{5}$, inter-particle collisions become the dominant source of dissipated energy despite there still being a greater proportion of particle-wall collisions. With $10^{6}$ particles, inter-particle collisions are now dominant in terms of number and energy loss.

Table 4. Change in dominant collision type with number of particles

\begin{tabular}{|c|c|c|c|}
\hline $\begin{array}{c}\text { No of } \\
\text { particles }\end{array}$ & $\mathbf{1 0}^{\mathbf{4}}$ & $\mathbf{1 0}^{\mathbf{5}}$ & $\mathbf{1 0}^{\mathbf{6}}$ \\
\hline $\begin{array}{c}\text { Total no of } \\
\text { collisions }\end{array}$ & $11 \times 10^{6}$ & $48 \times 10^{6}$ & $642 \times 10^{6}$ \\
\hline $\begin{array}{c}\text { Inter- } \\
\text { particle (\%) }\end{array}$ & 3.5 & 40.8 & 89.5 \\
\hline $\begin{array}{c}\text { Particle-wall } \\
\text { (\%) }\end{array}$ & 96.5 & 59.2 & 10.5 \\
\hline $\begin{array}{c}\text { P-P energy } \\
\text { (\%) }\end{array}$ & 4.1 & 71.2 & 97 \\
\hline $\begin{array}{c}\text { P-W energy } \\
\text { (\%) }\end{array}$ & 95.9 & 28.8 & 3 \\
\hline
\end{tabular}




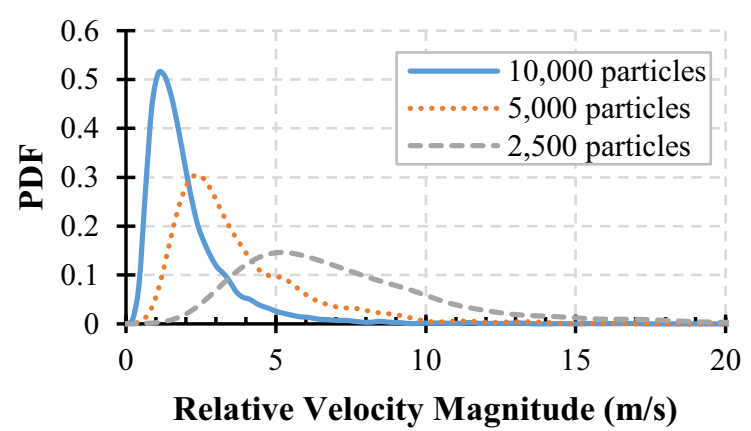

Fig. 6. Relative velocity distribution at $[4.0,4.0]$ for different number of particles

Increasing the number of particles in the spiral jet mill leads to a decrease in the relative velocity between particles (Fig. 6). It can also be seen that the relative velocity distribution widens as the particle number in the spiral jet mill reduces. The higher relative velocity leads to a greater degree of dissipated energy and subsequent particle breakage.

\subsection{Effect of Gas Pressure}

With $10^{4}$ particles, there is an increase in the particle velocity magnitude from $2.0-3.5 \mathrm{~m} / \mathrm{s}$ as the grinding pressure increases (Fig. 7). From gas pressure of 4.0 bar, increasing the pressure has an insignificant effect on the relative velocity magnitude suggesting that the particle concentration is too dense to have a significant effect on breakage. The relative velocity distribution also widens as the gas pressure increases.

Reducing the number of particles to 5,000 leads to a greater increase in relative velocity magnitude with gas pressure, from $2.5-5.5 \mathrm{~m} / \mathrm{s}$ (Fig. 8). This is due to particles having a greater amount of space in the chamber to accelerate and reach higher velocities before impact.

\section{Conclusions}

The spiral jet mill was simulated using DEM coupled with CFD to investigate the effect of different parameters on the energy transfer within the mill. Inter-particle collisions are found to have a higher dissipated collisional energy compared to particle-wall collisions, and therefore

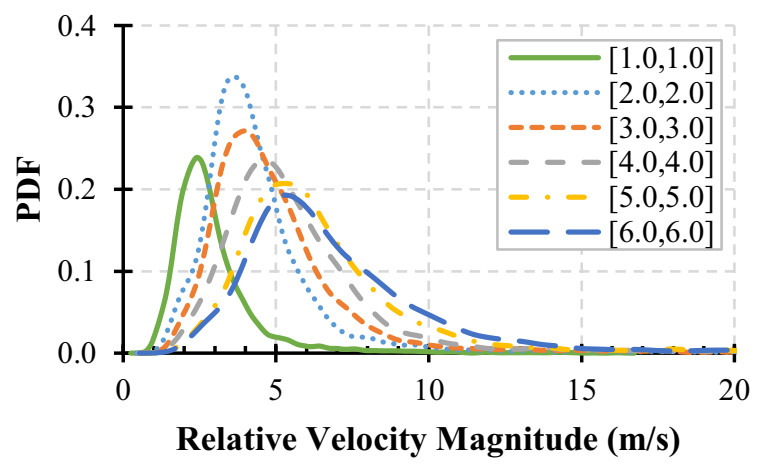

Fig. 7. Velocity distribution of $10^{4}$ particles

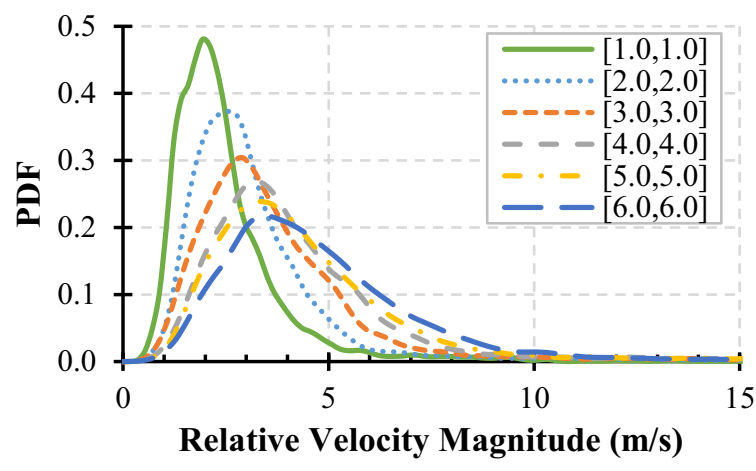

Fig. 8. Velocity distribution of 5,000 particles

expected to contribute more towards particle breakage. Collisions between 10 and $20 \mu \mathrm{m}$ particles are the most frequent ones and are expected to contribute most to particle breakage compared to other possible collisions, also influenced by particle size segregation within the grinding chamber. Particle concentration influences the degree of inter-particle and particle-wall collisions, with the former becoming more dominant than the latter in denser systems. Increasing the particle concentration also leads to narrower relative velocity distributions between colliding particles, as well as generally smaller relative velocity magnitudes. For dense systems, increasing the grinding pressure has some effect on the relative velocity distribution, but to a lesser extent on the general velocity magnitude of the collisions.

\section{Acknowledgements}

The financial support in the form of an Industrial CASE Award by the EPSRC and AstraZeneca (Macclesfield, $\mathrm{UK}$ ) for PhD studentship for the first author is gratefully acknowledged.

\section{References}

1. M. Metboul, J.F. Large, P. Guigon, Int. J. Miner. Process. 44-45 (1996)

2. T. Han, H. Kalman, A. Levy, Part. Sci. Technol. 20 (2002)

3. A. Levy, H. Kalman, Part. Sci. Technol. 25 (2007)

4. T. Brosh, Y. Batat, H. Kalman, A. Levy, A.B. Brown, Bulk Solids Powder - Sci. Technol. 3 (2008)

5. S. Teng, P. Wang, Q. Zhang, C. Gogos, Powder Technol. 208 (2011)

6. M. Pasha, S. Dogbe, C. Hare, A. Hassanpour, M. Ghadiri, Granul. Matter 16 (2014) 